\title{
Johnsonella ignava
}

National Cancer Institute

\section{Source}

National Cancer Institute. Johnsonella ignava. NCI Thesaurus. Code C86448.

A species of obligately anaerobic, Gram negative, rod shaped bacteria assigned to the phylum Firmicutes. This species is nonmotile, indole, oxidase and catalase negative, and nonfermentative. J. ig nava is commonly found in the ging ival crevices of the oral cavity and may cause ging ivitis and periodontitis. 